\title{
11 The phenomena of auditory verbal hallucinations and the role of Functional MRi: a systematic review
}

J. Pereira $^{1}$, D. Pereira ${ }^{1}$, G. Marinho ${ }^{1}$, T. Teodoro ${ }^{1}$, V. Viveiros ${ }^{1}$.

${ }^{1}$ Centro Hospitalar Psiquiátrico de Lisboa, Psychiatry, Lisboa, Portugal.

\section{Introduction:}

The Auditory-Verbal Hallucinations (AVH) are a core feature of schizophrenia spectrum disorders, having a disruptive effect on patient functionality. Furthermore, $\mathrm{AVH}$ is associated with higher rates of depression and suicide. It is crucial to fully understand how the symptom develops in order to promote target therapies, with higher efficiency and fewer secondary effects. Although the aetiology of the symptom is still unknown, the advance of functional MRi brought new and relevant data about the origins of $\mathrm{AVH}$.

\section{Objectives:}

Based on the advance of functional MRi research, we aim to collect the new data and expose the advances in the AVH knowledge.

\section{Methods:}

The present study is based on an extensive review of literature through Pubmed research motor, using the key-words "functional MRl" and "hallucinations". We obtained a total of 36 articles, of which excluded 24 for not being written in English or for not fit into our objectives.

\section{Results:}

The majority of the works appoint auditory cortex and speech processing regions as critical substrates for $\mathrm{AVH}$ generation, although many other areas seem to be active while $A V H$ is occurring as left and right inferior frontal and superior temporal gyri, right middle temporal gyrus, left insula, supramarginal gyrus, inferior parietal lobule, and extranuclear white matter.

\section{Results:}

According to Thoma et al. AVH results from an erroneous placement of a "stamp" of external generation on internal speech such that this internal dialog is perceived as having an outside origin.

On the other side, some authors as Wu et al. suggest $\mathrm{AVH}$ is correlated "with a reduction in the typical pattern of left-greater-than-right lateralization of language processing" 6 . In fact, the results from $W u$ et al. work do show "that both functional lateralization of the dorsal pathway and microestrutural integrity of the right dorsal pathway were negatively correlated with the scores of the delusion/hallucination symptom dimension" 6 .

At last, the dysconnectivity hypothesis suggests that AVH occur due to impaired connectivity between different brain regions. In fact, functional connectivity is altered within the language network of patients suffering AVH. Further more, white matter connectivity is impaired in pathways connecting the perception and production speech areas in schizophrenia patients with $\mathrm{AVH}$.

\section{Conclusions:}

Although it's clear the great importance of the auditory cortex and speech processing regions in the AVH generation, we still don't fully understand the specific type of aberrant connection between these two areas and the role of the other brain areas activated during $\mathrm{AVH}$.

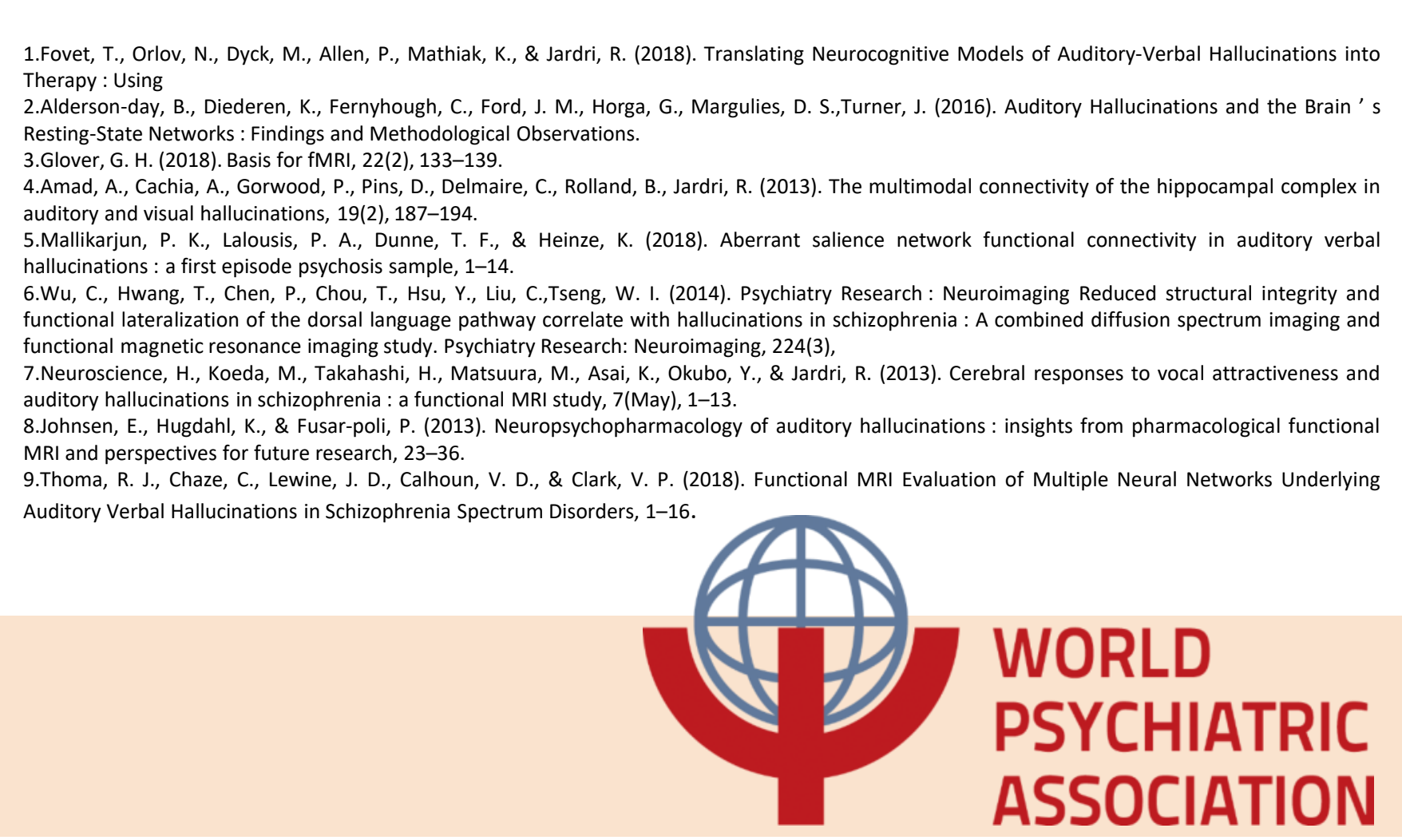

